sterilization process is recommended. For monitoring proper steam penetration inside hollow or complex instruments (ie, luminal instruments), HPCD is clearly the best option. ${ }^{10}$

\section{References}

1. Basu D, Bhattacharya S, Mahajan A, Ramanan VR, Chandy M. The importance of the central sterile supply department in infection prevention and control. Infect Control Hosp Epidemiol 2014;35:1312-1314.

2. Basu D, Bhattacharya S, Mahajan A, Ramanan VR, Chandy M. Sterilization indicators in central sterile supply department: quality assurance and cost implications. Infect Control Hosp Epidemiol 2015;36:484-486.

3. McDonnell G, Burke P. Disinfection: is it time to reconsider Spaulding? J Hosp Infect 2011;78:163-170.

4. Steri-record direct inoculation syringe to test sterility inside complex instruments. gke website. https://www.gke.eu/en/product-cataloguesand-data-sheets.html?file=files/gke_2013/content/en/downloads/Data\% 20sheets/Biological\%20indicators/Direct\%20Inoculation\%20Syringe.pdf. Published 2013. Accessed April 18, 2019.

5. Current reference devices for hollow instruments loads as defined in standards are not a valid steam penetration test. gke website. http://www.gke.de/en/publications.html?file=files/gke_2013/content/en/ downloads/Publications/Others/Current\%20reference\%20devices\%20for \%20hollow\%20instruments\%20loads.pdf. Published 2013. Accessed May 11, 2018.

6. Effectiveness of hospital central sterilization processing vs clinic-based sterilization protocols: a noninferiority trial. Dalhousie University Library website. https://dalspace.library.dal.ca/bitstream/handle/10222/ 54542/Stefanuto-Peter-MSc-OMFS-September-2014.pdf?sequence $=5$. Published 2014. Accessed May 11, 2018.

7. Lobdell KW, Stamou S, Sanchez JA. Hospital-acquired infections. Surg Clin N Am 2012;92:65-77.

8. Sasmal PK, Mishra TS, Rath S, Meher S, Mohapatra D. Port site infection in laparoscopic surgery: a review of its management. World J Clin Cases 2015;3:864-871.

9. Cost comparison of medical treatments: India vs the world. India Profile Medical Tourism website. http://www.indiaprofile.com/medical-tourism/ cost-comparison.html. Accessed April 10, 2018.

10. Performance of hollow load process challenge devices (HLPCDs) for the determination of air removal and steam penetration in porous load steam sterilization processes. University of Glascow Enlighten website. http://eprints.gla.ac.uk/136299/1/136299.pdf. Accessed May 10, 2018.

\title{
Serotype 14 pneumococcal bacteremia: From one neonate to another in a pediatric intensive care unit
}

\author{
Audrey Vincent $\mathrm{MD}^{1}$, Stéphane Bonacorsi MD, $\mathrm{PhD}^{2,3}$, Emmanuelle Varon $\mathrm{MD}^{4}$, Stéphane Dauger MD, $\mathrm{PhD}^{1,3}$ and \\ Michael Levy MD, PhD ${ }^{1,3}$ (i) \\ ${ }^{1}$ Pediatric Intensive Care Unit, Robert-Debré University Hospital, Assistance Publique Hôpitaux de Paris, Paris, France, ${ }^{2}$ Microbiology Unit, Robert-Debré \\ University Hospital, Assistance Publique Hôpitaux de Paris, Paris, France, ${ }^{3}$ University Paris Diderot Sorbone Paris Cité, Paris, France and ${ }^{4}$ National Reference \\ Centre for Pneumococci, Centre Hospitalier Intercommunal, Créteil, France
}

To the Editor-Streptococcus pneumoniae is a leading cause of community-acquired bacterial infections, but an unusual source of neonatal sepsis ${ }^{1}$ and nosocomial cases have rarely been described. We report 2 original cases of neonatal S. pneumoniae infection due to the same serotype 14 pneumococcal strain that occurred in the same pediatric intensive care unit (PICU) with a highly probable nosocomial contamination from one to the other.

Patient 1 was a 13-day-old boy admitted to the PICU for respiratory distress due to respiratory syncytial virus (RSV)-induced bronchiolitis on November 11. He developed apnea with bradycardia and a deep decrease in blood oxygen requiring noninvasive ventilation using bilevel positive airway pressure. His respiratory distress worsened 24 hours after admission due to left pulmonary pneumoniae, and a blood culture was positive with a serotype 14 S. pneumoniae isolate with decreased susceptibility to penicillin G (Table 1) and resistance to macrolides and cotrimoxazole. The patient was treated with cefotaxime $(100 \mathrm{mg} / \mathrm{kg} /$ day $)$. Evolution was favorable with spontaneous ventilation on day 4 , and he was discharged from the PICU on day 5 (November 15).

Author for correspondence: Michael Levy, Pediatric Intensive Care Unit, Robert-Debré University Hospital, 48, boulevard Sérurier, 75019 Paris, France. Email: Michael.levy@aphp.fr Cite this article: Vincent A, et al. (2019). Serotype 14 pneumococcal bacteremia: From one neonate to another in a pediatric intensive care unit. Infection Control \& Hospital Epidemiology, 40: 952-953, https://doi.org/10.1017/ice.2019.152
Patient 2 was a 18-day-old healthy full-term boy also admitted to the PICU for respiratory distress due to RSV-induced bronchiolitis on November 9. He had a right superior lobe pulmonary atelectasis, no signs of bacterial infection, and negative blood culture. Noninvasive ventilation using continuous positive airway pressure was performed, followed 2 days later by invasive ventilation because of the onset of acute respiratory distress syndrome (ARDS). On November 15, his clinical state worsened, with bilateral pneumonia, and a tracheal sample yielded $S$. pneumoniae with the same pattern of antibiotic susceptibility as case 1. Central line insertion, prone positioning, high-frequency ventilation, nitric oxide, corticotherapy, and antibiotic treatment with cefotaxime $(100 \mathrm{mg} / \mathrm{kg} /$ day for 5 days) were performed. Fever stopped 2 days after the initiation of antibiotherapy, and the patient was removed from mechanical ventilation on November 22. Fever reappeared on November 24 due to central-line-associated bloodstream infection (CLABSI). Streptococcus pneumoniae was detected in qualitative peripheral and catheter blood cultures performed November 25, as well as in a quantitative catheter central-line blood culture with $10^{4}$ colony-forming units $(\mathrm{CFU}) / \mathrm{mL}$. The catheter culture after removal was positive, with $10^{6} \mathrm{CFU} / \mathrm{mL}$. This serotype 14 $S$. pneumoniae harbored the same profile of susceptibility to antibiotics as case 1 (Table 1). Cefotaxime $(150 \mathrm{mg} / \mathrm{kg} /$ day) was introduced and continued for a total duration of 7 days after the first sterile blood culture. The patient's fever disappeared, and he was discharged from the PICU on November 27. Considering the antibiotic susceptibility 
Table 1. Characteristics of Streptococcus pneumoniae Strains

\begin{tabular}{lcc}
\hline Original Serotype & $\begin{array}{c}\text { Patient 1 Strain } \\
\text { Blood Culture } \\
(\mathrm{n}=14)\end{array}$ & $\begin{array}{c}\text { Patient 2 Strain } \\
\text { Blood Culture } \\
(\mathrm{n}=14)\end{array}$ \\
\hline Genotype & 7 & 7 \\
\hline -aroE & 11 & 11 \\
\hline -gdh & 10 & 10 \\
\hline -gki & 1 & 1 \\
\hline -recP & 6 & 6 \\
\hline -spi & 8 & 8 \\
\hline -xpt & 267 & 267 \\
\hline -ddl & 2,918 & 2,918 \\
\hline Sequence type & 156 & 156 \\
\hline Clonal complex & & 1 \\
\hline Antibiotic susceptibility & 1 & 1 \\
\hline - MIC to penicillin & 1 & 0.25 \\
\hline - MIC to amoxicillin & 0.25 & 0.25 \\
\hline - MIC to cefotaxime & 0.25 & \\
\hline - MIC to ceftriaxone & & \\
\hline
\end{tabular}

Note. MIC, minimum inhibitory concentration.

pattern, the serotype of the isolates of both cases, and the fact that both neonates were cared for by the same caregivers, multilocus sequence typing (MLST) was performed on the S. pneumoniae detected in blood cultures of both patients. Both strains were of the same ST2918 belonging to the clonal complex (CC) 156 (Table 1).

Reports of large series of neonatal sepsis in the post-penicillin era have shown that $S$. pneumoniae is a rare cause of neonatal infection $(1 \%-11 \%) .{ }^{1,2}$ The predominant clinical presentation in neonates is bacteremia, but other clinical manifestations have been reported. ${ }^{3}$ We report here 2 cases of $S$. pneumoniae infection occurring in the same PICU in 2 neonates, including 1 CLABSI. Both infections were due to a serotype 14 S. pneumoniae, a serotype included in the 7-valent pneumococcal conjugate vaccine (PCV7) introduced in France in 2003. PCV7 introduction resulted in a dramatic decrease of PCV7 invasive pneumococcal disease (IPD). Serotype 14 represented 15\% of IPD in 2001-2002 but represented $<1 \%$ after 10 years. ${ }^{4}$ Although most infants $<60$ days of age are too young to receive any direct protection from vaccination, it has been observed in the United States and England that they benefited from PCV7 through indirect (herd) protection., ${ }^{5,6}$ This herd protection also resulted in a drastic decrease of $S$. pneumoniae included in the PCV7 nasopharyngeal carriage (-97\%) in children. ${ }^{7}$ Therefore, IPD due to serotype 14 in neonates should be exceptional, and the occurrence of 2 infections due to this serotype in our ICU in $<12$ days strongly suggests a nosocomial transmission. Although whole-genome sequencing of the 2 strains was not performed, the sequence type determination revealed a single locus variant of ST156 (CC156) that corresponded to the highly successful clone Spain ${ }^{9 \mathrm{~V}}-3 .{ }^{8}$ Interestingly, ST2918 is a very rare sequence type among the CC156, and only 1 strain among 2,266 serotype 14 S. pneumoniae isolates available on the PubMLST public databases (https://pubmlst.org/spneumoniae/) belonged to ST2918. Thus, considering the rarity of IPD of serotype 14 and the rarity of ST2918 among serotype 14 S. pneumoniae, it is highly probable that healthcare transmission of the pathogen occurred between the two patients.

In addition, nosocomial pneumococcal bloodstream infections (NPBI) are rarely described in the literature, ${ }^{9}$ with pneumonia being the major portal of entry (70.1\%), whereas CLABSI represented only $3.9 \%$. Therefore, our second case is of particular interest; it demonstrates that although CLABSI due to S. pneumoniae is rare, ${ }^{9,10}$ clinicians should be aware of this potential source of bacteremia.

In conclusion, we report 2 original cases of neonatal S. pneumoniae infection with a highly probable healthcare-associated contamination from one patient to another. These 2 infections were likely due to a single serotype $14 \mathrm{~S}$. pneumoniae strain in neonates that were too young to receive pneumococcal conjugate 13-valent vaccine, and one of these patients developed secondary CLABSI.

Author ORCIDs. Michael Levy (iD) 0000-0003-2766-5777

Financial support. No financial support was provided relevant to this article.

Conflicts of interest. All authors report no conflicts of interest relevant to this article.

\section{References}

1. Hoffman JA, Mason EO, Schutze GE, et al. Streptococcus pneumoniae infections in the neonate. Pediatrics 2003;112:1095-1102.

2. Westh H, Skibsted L, Korner B. Streptococcus pneumoniae infections of the female genital tract and in the newborn child. Clin Infect Dis 1990;12: 416-422.

3. Kaplan M, Rudensky B, Beck A. Perinatal infections with Streptococcus pneumoniae. Am J Perinatol 1993;10:1-4.

4. Batah J, Varon E. Rapport d'activité du CNR des Pneumocoques 2017. CNRP website. http://cnr-pneumo.com/docs/rapports/CNRP2017. pdf. Published 2017. Accessed May 21, 2019.

5. Ladhani SN, Andrews NJ, Waight P, Borrow R, Slack MPE, Miller E. Impact of the 7-valent pneumococcal conjugate vaccine on invasive pneumococcal disease in infants younger than 90 days in England and Wales. Clin Infect Dis 2013;56:633-640.

6. Soto-Noguerón A, Carnalla-Barajas MN, Solórzano-Santos F, et al. Streptococcus pneumoniae as cause of infection in infants less than 60 days of age: serotypes and antimicrobial susceptibility. Int J Infect Dis 2016; 42:69-73.

7. Cohen R, Biscardi S, Levy C. The multifaceted impact of pneumococcal conjugate vaccine implementation in children in France between 2001 and 2014. Hum Vaccines Immunother 2016;12:277-284.

8. McGee L, McDougal L, Zhou J, et al. Nomenclature of major antimicrobialresistant clones of streptococcus pneumoniae defined by the pneumococcal molecular epidemiology network. J Clin Microbiol 2001;39:2565-2571.

9. Bouza E, Pintado V, Rivera S, et al. Nosocomial bloodstream infections caused by Streptococcus pneumoniae. Clin Microbiol Infect 2005;11:919-924.

10. Canet J, Juan N, Xercavins M, Freixas N, Garau J. Hospital-acquired pneumococcal bacteremia. Clin Infect Dis 2002;35:697-702. 